\title{
Validation of proton ionization cross section generators for Monte Carlo particle transport
}

\author{
Matej Batič, Maria Grazia Pia, Paolo Saracco
}

\begin{abstract}
Three software systems, ERCS08, ISICS 2011 and Šmit's code, that implement theoretical calculations of inner shell ionization cross sections by proton impact, are validated with respect to experimental data. The accuracy of the cross sections they generate is quantitatively estimated and inter-compared through statistical methods. Updates and extensions of a cross section data library relevant to PIXE simulation with Geant4 are discussed.
\end{abstract}

Index Terms-Monte Carlo, simulation, Geant4, ionization, PIXE

\section{INTRODUCTION}

$\mathbf{T}$ HE calculation of inner shell ionization cross sections by proton and ion impact is an important component of the simulation of PIXE (Particle Induced X-ray Emission) and the analysis of experimental PIXE spectra. The ECPSSR (Energyloss Coulomb Perturbed Stationary State Relativistic) [1] theory with its variants is regarded as the standard approach for cross section calculations in the domain of PIXE applications, which typically concern the energy range up to a few tens $\mathrm{MeV}$ and the whole range of elements in the periodic system. It provides inner shell ionization cross sections for PIXE analysis codes such as GeoPIXE [2], GUPIX [3]-[5], PIXAN [6], PIXEF [7], PIXYKLM [8], Sapix [9], and TTPIXAN [10], and for specialized PIXE simulation codes [11]-[13].

Several cross section models for the computation of inner shell ionization by proton and $\alpha$ particle impact are available in a package for PIXE simulation [14] released in Geant4 [15], [16] 9.4 version; they include models based on the plane wave Born approximation (PWBA) [17], the ECPSSR model in a number of variants and a collection of empirical models, deriving from fits to experimental data. The PWBA and ECPSSR cross section models (with variants) exploit tabulations produced by the ISICS [18] code for K, L and M shells, which have been assembled in a data library [19] publicly distributed by RSICC (Radiation Safety Information Computational Center at the Oak Ridge National Laboratory).

A new version of ISICS and an entirely new code for the calculation of ECPSSR cross sections (with variants), ERCS08 [20], have become available since the publication of the previously cited paper on Geant4 PIXE simulation. This paper evaluates the cross sections deriving from these

Manuscript received May 18, 2011.

M. G. Pia and P. Saracco are with INFN Sezione di Genova, Via Dodecaneso 33, I-16146 Genova, Italy (phone: +39 010 3536328, fax: +39 010 313358, MariaGrazia.Pia@ge.infn.it, saracco@ge.infn.it).

M. Batič is with INFN Sezione di Genova, Genova, Italy (e-mail: Matej.Batic@ge.infn.it); he is on leave from Jozef Stefan Institute, 1000 Ljubljana, Slovenia. evolutions, along with those calculated by Šmit's code [21] (identified in the following as KIO-LIO), which is consistent with pristine ECPSSR formulation.

The $\mathrm{K}$ and $\mathrm{L}$ shell proton ionization cross sections produced by these three theoretical generators are compared with reference collections of experimental data to assess their validity, in compliance with the IEEE Standard for Software Verification and Validation [22]. The results of this validation process document quantitatively the relative merits of the three codes, evaluate the impact of the newly available calculations on Geant 4 accuracy and identify the state-of-the-art of theoretical cross sections for PIXE simulation with Geant4.

\section{THEORETICAL OVERVIEW}

In the PWBA approach [17], the first-order Born approximation is used in scattering theory to describe the interaction between an incident charged particle and an atomic target. This treatment is justified when the atomic number of the projectile is much smaller than the atomic number of the target, and the velocity of the incident particle is much larger than the velocity of the target-atom electron velocities.

The PWBA cross section in the center of mass system for the ionization of a given shell is given by

$$
\sigma_{\mathrm{PWBA}}=\sigma_{0} \theta^{-1} F\left(\frac{\eta}{\theta}, \theta\right)
$$

where:

$$
\sigma_{0}=8 \pi a_{0}^{2}\left(\frac{Z_{1}^{2}}{Z_{2}^{4}}\right)
$$

$a_{0}$ is the Bohr radius, $Z_{1}$ is the projectile atomic number, $Z_{2}$ is the effective atomic number of the target atom, $F$ is the reduced universal function, and the reduced atomic electron binding energy and reduced projectile energy are given by

$$
\begin{gathered}
\theta=2 n^{2} \frac{U_{2}}{Z_{2}^{2}} \\
\eta=2 \frac{E_{1}}{M_{1} Z_{2}^{2}}
\end{gathered}
$$

with $\mathrm{E}, \mathrm{M}$ and $\mathrm{U}$ representing the energy, mass and atomic binding energy of the projectile and the target, respectively identified by the indices 1 and 2 . The analytical formulation of the reduced universal function $F$ can be found in [18].

The ECPSSR theory [1] was proposed to address the shortcomings of the PWBA approach in the energy range relevant to PIXE experimental practice (approximately up to a few tens of $\mathrm{MeV}$ ); it accounts for the energy loss and Coulomb deflection of the projectile, the perturbed stationary state and relativistic 
nature of the target's inner shell. The ECPSSR cross section for a given shell is expressed in terms of the PWBA one:

$$
\sigma_{\mathrm{ECPSSR}}=C_{B}^{E}\left(d q_{0}^{B} \zeta\right) \sigma_{\mathrm{PWBA}}\left(\frac{m_{R}\left(\frac{\xi}{\zeta}\right) \eta}{(\zeta \theta)^{2}}, \zeta \theta\right)
$$

where $C_{E}^{B}$ is the Coulomb deflection correction, $\zeta$ is the correction factor for binding energy and polarization effects, $m_{R}$ is the relativistic correction, $q_{0}$ is the minimum momentum transfer and

$$
\xi=v_{1} \frac{Z_{2}}{U_{2}}
$$

$v_{1}$ being the projectile velocity.

Further refinements and modifications of the ECPSSR theory have been proposed: they involve using relativistic DiracHartree-Slater wave functions in computing the form factors of the theory [24] (identified in the following as ECPSSR-HS), account for changes in binding energy of electron states due to the presence of the positive ion projectile in the atom (united atom correction, identified as ECPSSR-UA), and extend the applicability of the theory to relativistic light projectiles for $\mathrm{K}$ shell calculations [25] (identified as ECPSSR-HE).

\section{CROSS SECTION GENERATORS}

The evaluation concerns three publicly accessible generators of proton ionization cross sections. Two of them, ISICS and ERCS08, are distributed through the Computer Physics Communications (CPC) Program Library; the third is available directly from its author, as specified in [21].

\section{A. ISICS}

The ISICS code [18], [26], [27] calculates K, L and M shell ionization cross sections by proton and $\alpha$ particle impact according to the PWBA approximation and the ECPSSR theory in multiple variants.

The first version of ISICS [18] provided the options of PWBA and plain ECPSSR calculations; later versions have added capabilites for Hartree-Slater calculations [26], united atom option [23] and treatment of relativistic light projectiles [27]. The latest version distributed through the CPC Program Library at the time of writing this paper is ISICS 2011 [28]; it implements a few changes with respect to the previous 2008 version, that contribute to the numerical robustness of the code.

ISICS versions up to 2008 used Bearden and Burr's compilation [29] of atomic binding energies; ISICS 2011 version provides also the option of using Williams' compilation [30], [31], although the default configuration still uses Bearden and Burr's values.

The theoretical cross sections included in the PIXE data library associated with Geant 49.4 have been produced with ISICS 2008.

\section{B. ERCSO8}

ERCS08 [20] is a FORTRAN program for computing electron removal cross section by protons and heavier ions. It encompasses the calculation of direct ionization and electron capture cross sections; only the former is considered in this paper.

The calculations are based on the ECPSSR theory; the program allows the configuration of individual components of the theory (E, C, PSS, R), as well as other options: R-left (as described in [25]), united atom and hSR (as described in [24]).

The program provides a default configuration for each shell type (K, L or M), which can be overridden by the user. The default configuration for the $\mathrm{K}$ shell activates the calculation of the form factor involved in the ECPSSR theory using exact limits of integration and the correction for relativistic HartreeSlater wave functions for the K shell (the hSR option). The united atom approach is taken into account in the default configuration for all shells.

A few test cases documented in [20] show that ERCS08 calculates ECPSSR cross sections compatible with ISICS 2006 version to one unit at the fourth significant figure, when it is configured to reproduce the same plain ECPSSR settings as ISICS 2006. Although [20] states that differences with respect to ISICS may be larger when ERCS08 is run using its own default parameters and input data, the authors of this paper could not find evidence of the experimental validation of the default configuration of this code in the literature.

The code includes a set of atomic binding energies; according to [20], they derive from experimental values in the literature when available, otherwise from theoretical values or, when neither was deemed available, from a first order linear regression analysis applied to available data for elements with atomic number greater than 79 documented in [20]. Some of the binding energies distributed with ERCS08 could be identified as taken from Bearden and Burr's compilation [29], the theoretical values of Table IV in [32] and the 1978 edition of the Table of Isotopes [33]; however, the source of other values remains unidentified. Although theoretical binding energies are available for any elements with atomic number up to 100 in EADL (Evaluated Atomic Data Library) [34], they do not appear to have been used in the program.

\section{KIO-LIO}

A software system to calculate ECPSSR cross sections has been developed in Pascal by Ž. Šmit [21] and is available on request directly from its author; it can be executed on Windows systems. Since it does not appear identified in the literature by a specific name, for convenience it is referred to in the following as KIO-LIO, as these are the names of the executable files provided by the author respectively for $\mathrm{K}$ and L shell computations.

The code implements the method described in [21] for the $\mathrm{K}$ shell and $L_{1}, L_{2}$ subshells; the cross section for $L_{3}$ subshell ionization is calculated by the same function as that for the $L_{2}$ subshell, with appropriate binding energy value and further multiplied by a factor two.

The code includes three options of atomic electron binding energies, corresponding to the compilations by Bearden and Burr [29], the 1978 edition of the Table of Isotopes [33] and Sevier [35]; nevertheless, they cannot be selected through 
the user interface, therefore the distributed executable files limit the production to the default configuration with Sevier's binding energies.

\section{Comparative features of calculated cross sections}

All of the three generators implement calculations based on the ECPSSR theory; nevertheless, as it can be observed in Fig. 14 the cross sections they calculate exhibit some differences. The figures adopt consistent color, line and marker style throughout the paper to facilitate the identification of the behavior of each generator.

The histograms in Fig. 1 concern cross sections generated by ERCS08 and KIO in their default configurations for K shell, and by ISICS configured according to the same nominal options as the two other codes: to calculate ECPSSR-HS-UA cross sections (nominally equivalent to ERCS08 default) and plain ECPSSR ones (nominally equivalent to KIO). The cross sections are calculated at the same energies as the experimental data collected in [36], which, apart from a few measurements at $160 \mathrm{MeV}$, span the energy range up to approximately 50 $\mathrm{MeV}$.

Fig. 24 concerns L shell cross sections calculated at the same energies (up to $4 \mathrm{MeV}$ ) as the experimental data in [37], [38]: by ERCS08 and LIO in their default configurations for L shell, and by ISICS configured to calculate ECPSSR-UA cross sections (nominally equivalent to ERCS08 default for L subshells). The histograms of ISICS plain ECPSSR cross sections, corresponding to LIO's default configuration, are not superimposed to the other plots for better clarity, since they look very close to those produced by the ECPSSR-UA option.

For nominally equivalent theoretical approaches, discrepant results may arise from different mathematical methods adopted in the calculations, the use of different atomic parameters (e.g. binding energies), different software algorithms or numerical precision enforced in the code, or other computational details. Comparisons with experimental data are required to quantify how the differences of the various cross section generators affect the accuracy of the results they produce.

\section{VALIDATION METHOD}

The validation process involves the comparison of cross sections calculated by the three generators with experimental data.

\section{A. Experimental references}

The same sources of experimental data used in the validation of the Geant4 PIXE data library documented in [14] were exploited in the validation process of the three cross section generators considered in this paper. They are established compilations of experimental data in the field; moreover, the use of the same experimental references allows a comparative evaluation of the accuracy of the newly available generators and the currently distributed Geant4 PIXE data library, thus facilitating the identification of possible improvements to it.

The reference experimental data for $\mathrm{K}$ shell ionization were extracted from the compilation in [36], which includes

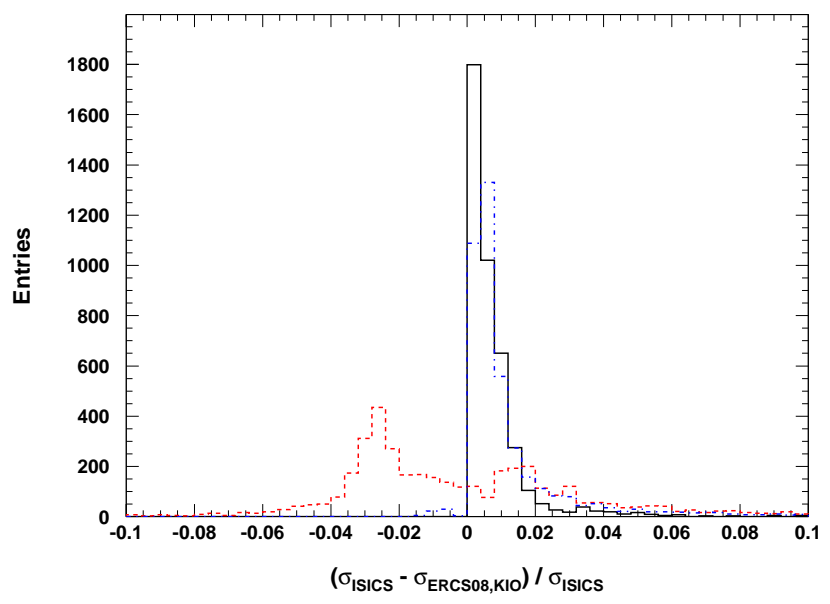

Fig. 1. K shell ionization: relative difference of cross sections calculated by default ERCSO8 configuration (solid black histogram) and KIO (dashed red histogram) with respect to ISICS ECPSSR-HS-UA configuration; relative difference of cross sections calculated by default KIO configuration with respect to ISICS plain ECPSSR configuration (dot-dashed blue histogram).

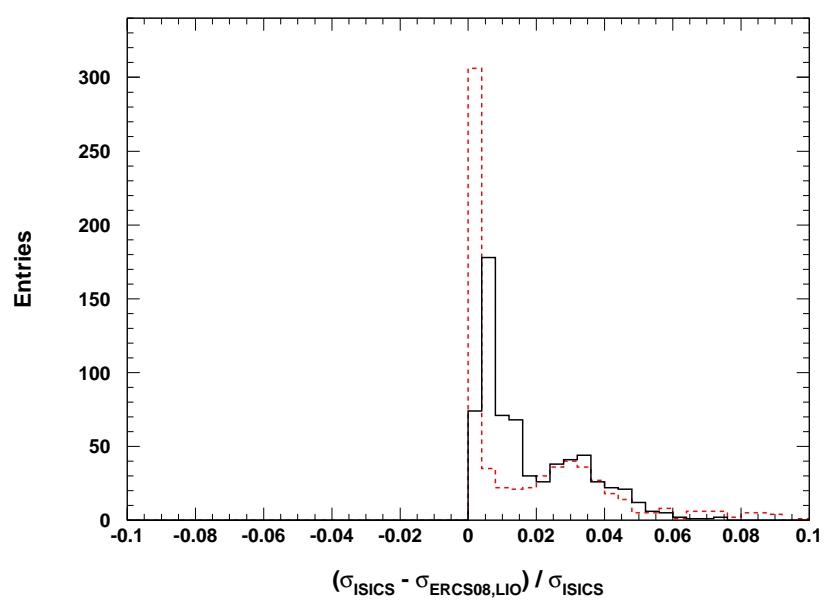

Fig. 2. $L_{1}$ subshell ionization: relative difference of cross sections calculated by default ERCS08 configuration (solid black histogram) and LIO (dashed red histogram) with respect to ISICS ECPSSR-UA configuration.

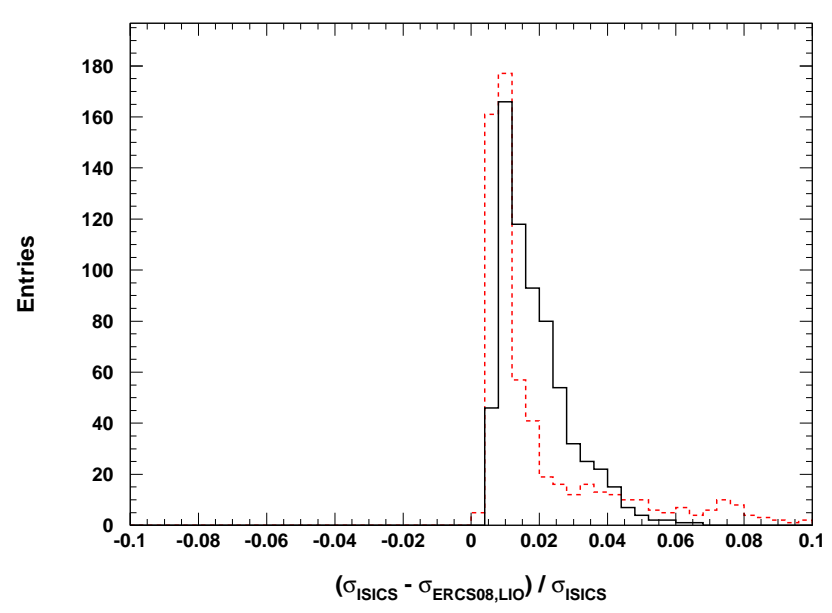

Fig. 3. $L_{2}$ subshell ionization: relative difference of cross sections calculated by default ERCS08 configuration (solid black histogram) and LIO (dashed red histogram) with respect to ISICS ECPSSR-UA configuration. 


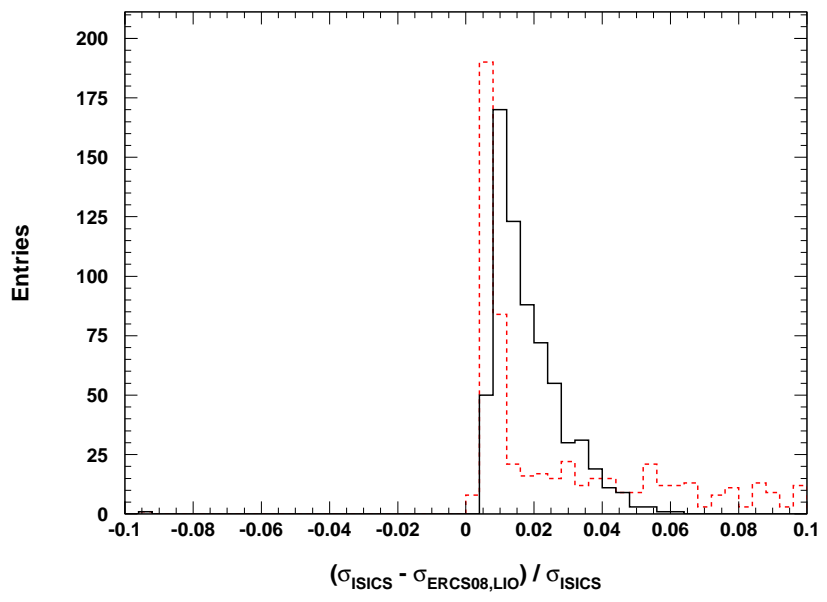

Fig. 4. $L_{3}$ subshell ionization: relative difference of cross sections calculated by default ERCS08 configuration (solid black histogram) and LIO (dashed red histogram) with respect to ISICS ECPSSR-UA configuration.

ionization cross sections along with X-ray and Auger electron production cross sections. The production cross sections were rescaled into ionization ones using the fluorescence yields compiled in [39]; uncertainties were propagated accordingly.

The reference data for $\mathrm{L}$ shell cross sections validation were assembled from two complementary collections [37], [38]. The experimental compilations report only total L shell cross section data for a few lighter elements; sub-shell data are listed only for elements with atomic number larger than 44. The experimental data often exhibit large discrepancies, and systematic effects are likely to be present for some elements, for which measurements deriving from different sources appear to be discordant.

\section{B. Theoretical cross section production}

ISICS cross sections were calculated using ISICSoo, a refactored version of the original ISICS Windows code, which has been especially tailored for large scale productions of data libraries on Linux platforms. ISICSoo provides the same physics functionality as ISICS 2011; its detailed features and verification are documented in [40]. The source code is intended to be available through the Computer Physics Communications Program Library.

The cross sections generated by ISICSoo appear equivalent to those computed by ISICS 2008; the relative differences, as shown for example in Fig. 5 for $\mathrm{K}$ shell ionization cross sections corresponding to plain ECPSSR configuration, are of the order of $10^{-5}$.

ERCS08 was compiled and run on a Linux system to produce the cross sections subject to validation. The production on a Linux platform required a small modification to the code released through the CPC Program library, limited to the user interface for run control. Input files were generated for each of the test cases through the Windows GUI to ensure consistency with the default settings provided by the system; they activated only the calculation of direct ionization. The ERCS08 production system in a Linux environment was

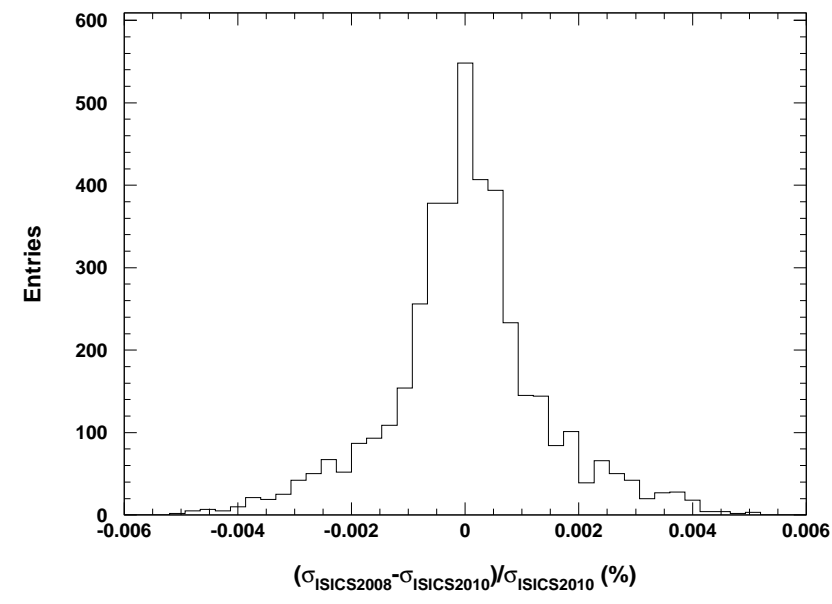

Fig. 5. Relative difference of $\mathrm{K}$ shell ionization cross sections calculated by ISICS 2008 and 2011 version with the plain ECPSSR option; the latter were produced by the refactored version of the code identified as ISICSoo. The cross sections were computed in both cases at incident proton energies and for target elements corresponding to the experimental data in [36].

verified to generate identical results to the original code run through the Windows GUI, consistent with those documented in [20].

The production of ISICS and ERCS08 cross sections was performed on an Intel Core2 Quad Q9300 CPU computer equipped with Scientific Linux 5 operating system and gcc 4.1.2 compiler.

The executable provided by the author was used for the production of KIO-LIO cross sections on a Microsoft Windows XP platform.

For the purpose of validation the theoretical cross sections were calculated at the same energy as the experimental data to avoid any perturbation to the intrinsic accuracy of the theoretical generators due to interpolation algorithms. This test configuration differs from the one reported in [14], where the cross sections subject to test were interpolated from tabulations produced according to the pre-defined energy grid adopted for the data library associated with the Geant 4 toolkit. The different configuration is justified by the different purpose of the test process: validating the Geant 4 cross section simulation models, that include the interpolation of a data library, in the previous paper, and evaluating the intrinsic accuracy of three theoretical generators in this one.

\section{Data analysis}

As remarked in the previous sections, the differences among the values calculated by the three generators are small (approximately a few percent); their effect on the accuracy of the cross sections can be appreciated only through statistical methods.

The analysis for the validation of theoretical cross section calculations follows the procedure reported in [14]. It is articulated over two stages: first a series of $\chi^{2}$ tests [41], comparing theoretical and experimental cross sections for each element and shell or subshell, followed by categorical analysis to identify differences across the results of the $\chi^{2}$ test associated with the three generators. Some experimental 


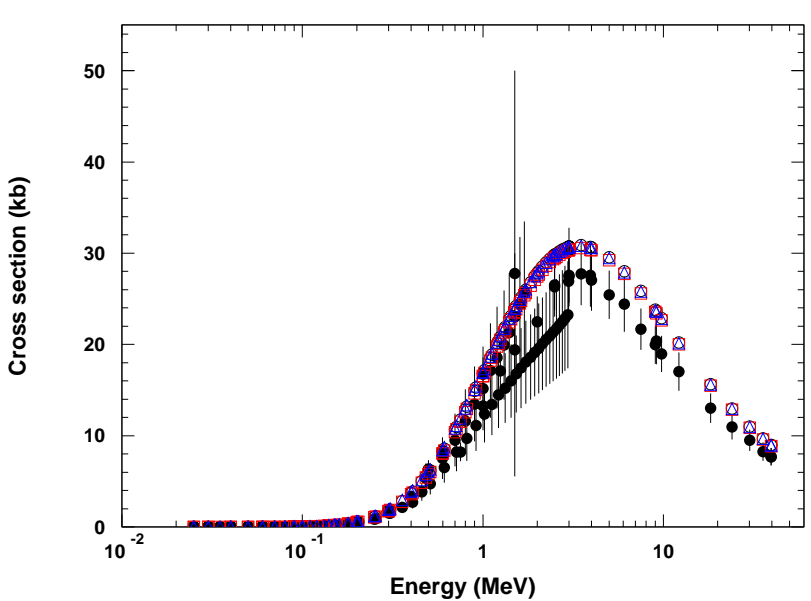

Fig. 6. K shell ionization cross sections by proton impact on aluminium calculated by theoretical generators and experimental data from [36] (black filled circles): ISICS 2011 in ECPSSR-HS-UA configuration (empty circles), ERCS08 (empty squares) and KIO (empty triangles), both in default configuration.

data exhibiting large discrepancies with respect to other data at the same or neighboring energies, suggesting the presence of systematic effects, were excluded from the computation of the $\chi^{2}$ statistic. The Statistical Toolkit [42], [43] was used for goodness-of-fit tests.

The null hypothesis in the goodness-of-fit testing process is defined as the compatibility that the theoretical and experimental cross section distributions subject to comparison derive from the same parent distribution. Unless differently specified, a 0.05 significance level is set to define the critical region of rejection of the null hypothesis.

Contingency tables are built from the outcome of the $\chi^{2}$ test to determine the equivalent behavior of the generators. The input to contingency tables derives from the results of the $\chi^{2}$ test for each element and shell or subshell: they are classified respectively as "pass" or "fail" according to whether the corresponding p-value is consistent with the defined significance level. The null hypothesis consists in assuming, for each contingency table, the equivalence of the generators it compares at reproducing experimental measurements. The contingency tables are analyzed with Fisher's exact test [44], with the $\chi^{2}$ test applying Yates' continuity correction [45], and with Pearson's $\chi^{2}$ test [46] when the number of entries in the cells justifies its applicability. A significance level of 0.05 is set to determine the rejection of the null hypothesis, unless specified differently.

\section{Results}

A selection of experimental data and cross sections calculated by the three generators is displayed in Fig. 66, the theoretical values correspond to the generators' configurations analyzed in the following sections.

A comprehensive overview of how theoretical cross sections compare to measurements is shown in Fig. 10,13, the plots display the difference between the cross sections calculated by the three generators and experimental data, divided by

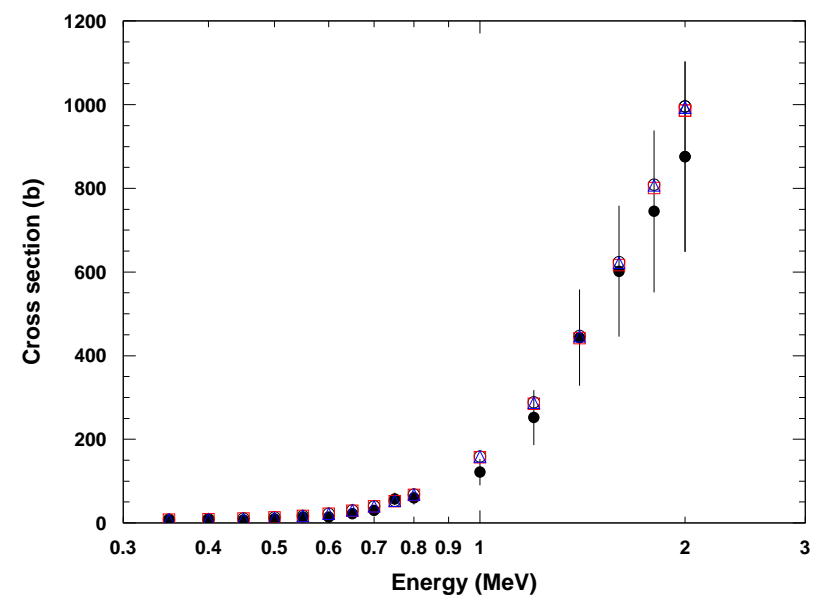

Fig. 7. $L_{1}$ subshell ionization cross sections by proton impact on tellurium calculated by theoretical generators and experimental data from [37], [38] (black filled circles): ISICS 2011 in ECPSSR-UA configuration (empty circles), ERCS08 (empty squares) and LIO (empty triangles), both in default configuration.

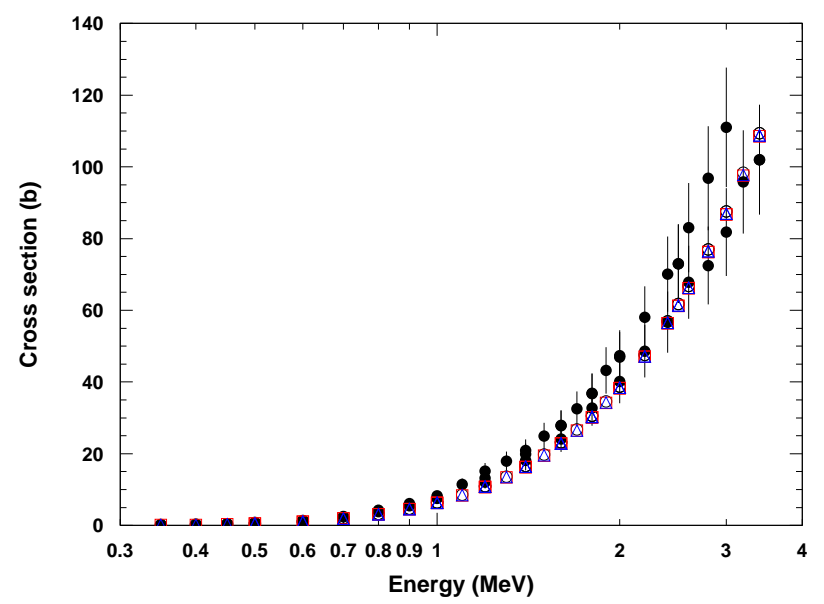

Fig. 8. $\quad L_{2}$ subshell ionization cross sections by proton impact on tungsten calculated by theoretical generators and experimental data from [37], [38] (black filled circles): ISICS 2011 in ECPSSR-UA configuration (empty circles), ERCS08 (empty squares) and LIO (empty triangles), both in default configuration.

the corresponding experimental uncertainties. In other words, they show by how many standard deviations experimental values differ from theoretical ones. These plots include all the experimental data of [36]-[38], without excluding any outliers. Theoretical generators appear to systematically underestimate $L_{1}$ and $L_{2}$ experimental cross sections.

The following sections report the statistical analysis to evaluate in detail the compatibility of the theoretical cross sections with experimental measurements. It is worthwhile to note that the incompatibility with experiment of all generators in some test cases hints to either an intrinsic deficiency of the underlying theory or to systematic effects in the experimental data. 


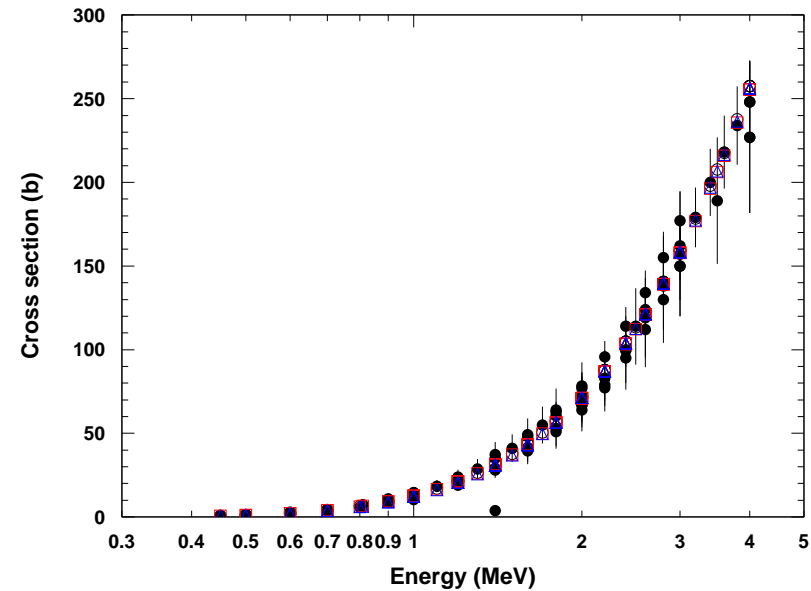

Fig. 9. $L_{3}$ subshell ionization cross sections by proton impact on gold calculated by theoretical generators and experimental data from [37], [38] (black filled circles): ISICS 2011 in ECPSSR-UA configuration (empty circles), ERCS08 (empty squares) and LIO (empty triangles), both in default configuration.

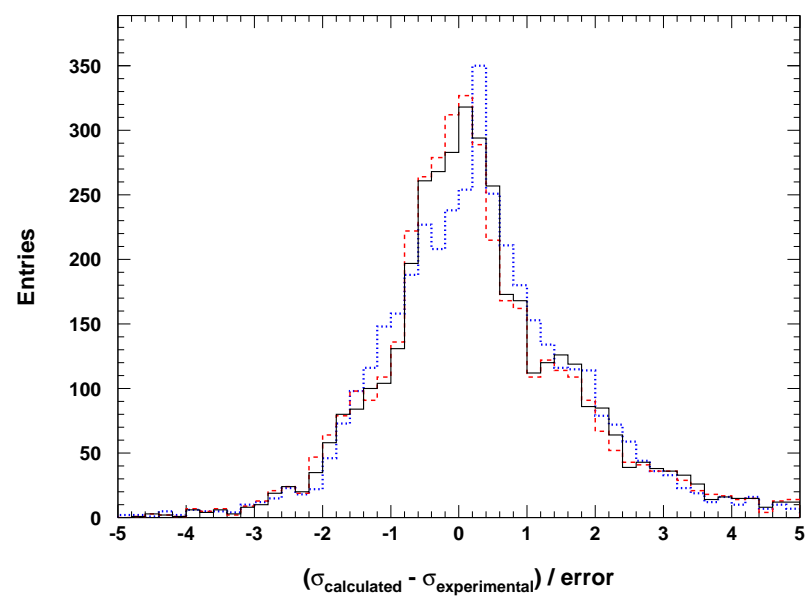

Fig. 10. Difference between $\mathrm{K}$ shell cross sections calculated by the three generators and experimental data, divided by the related experimental uncertainties: ISICS 2011 in ECPSSR-HS-UA configuration (solid black histogram), ERCS08 (red dashed histogram) and KIO (dotted blue histogram) in default configuration.

\section{A. K shell}

The p-values of the $\chi^{2}$ test comparing calculated and experimental $\mathrm{K}$ cross sections are reported for each tested element in Table I. The results are listed for ERCS08 and KIO in their default configuration, for ISICS 2011 in various options, two of which, ECPSSR-HS-UA and plain ECPSSR, are similar to the default configurations of the other codes.

The results are summarized in Table II where the test cases for which the null hypothesis of compatibility of calculated and experimental distributions is rejected at 0.05 significance level are counted as "fail", otherwise as "pass". The efficiency of each theoretical generator's configuration is defined as the ratio between the "passed" and total test cases.

It is evident from the results in Table II that ISICS 2011 ECPSSR-HS, ECPSSR-HS-UA and ERCS08 default configu-

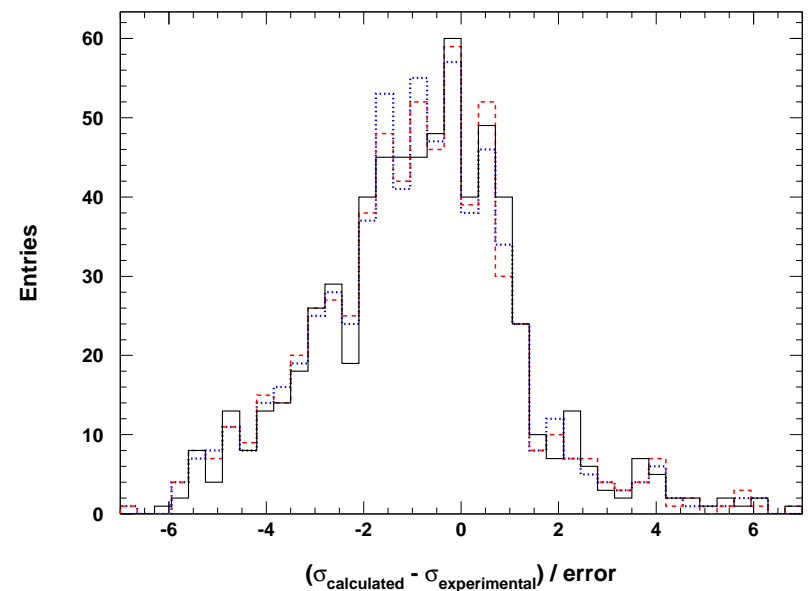

Fig. 11. Difference between $L_{1}$ subshell cross sections calculated by the three generators and experimental data, divided by the related experimental uncertainties: ISICS 2011 in ECPSSR-UA configuration (solid black histogram), ERCS08 (red dashed histogram) and LIO (dotted blue histogram) in default configuration.

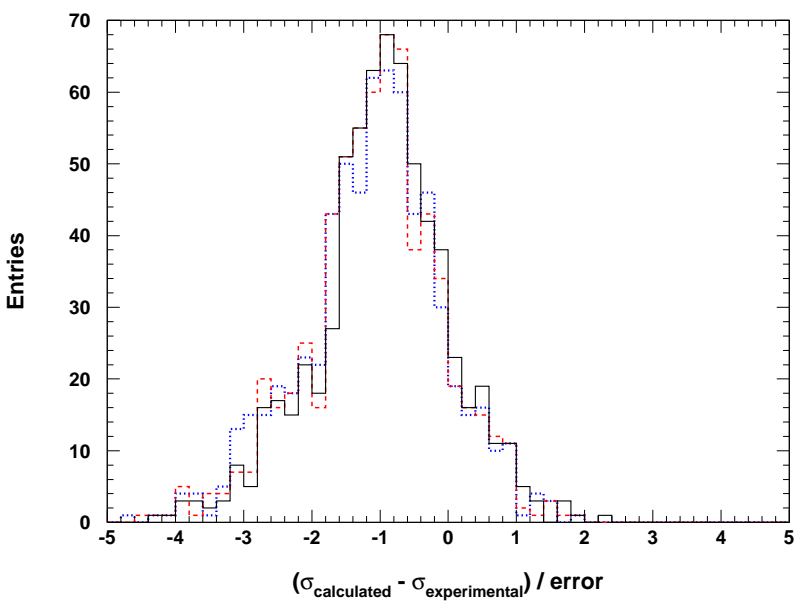

Fig. 12. Difference between $L_{2}$ subshell cross sections calculated by the three generators and experimental data, divided by the related experimental uncertainties: ISICS 2011 in ECPSSR-UA configuration (solid black histogram), ERCS08 (red dashed histogram) and LIO (dotted blue histogram) in default configuration.

ration are the most efficient at reproducing experimental cross sections, while KIO and the other ISICS options produce slightly less accurate results. Nevertheless, as shown in the contingency tables in Table III the differences in efficiency are not statistically significant at $95 \%$ confidence level.

Although the statistical analysis over the complete data samples provides an overall picture of the accuracy of the three cross section generators, the detailed results of the $\chi^{2}$ test in Table \ can be useful to optimize the choice in some specialized use cases: for instance, ERCS08 cross sections for silicon are compatible with experimental data [36], while the two other generators fail at reproducing compatible cross sections at 0.05 significance level for this element; KIO succeeds at reproducing equivalent tungsten cross sections at 0.05 significance level, while both ISICS ECPSSR-HS-UA and 


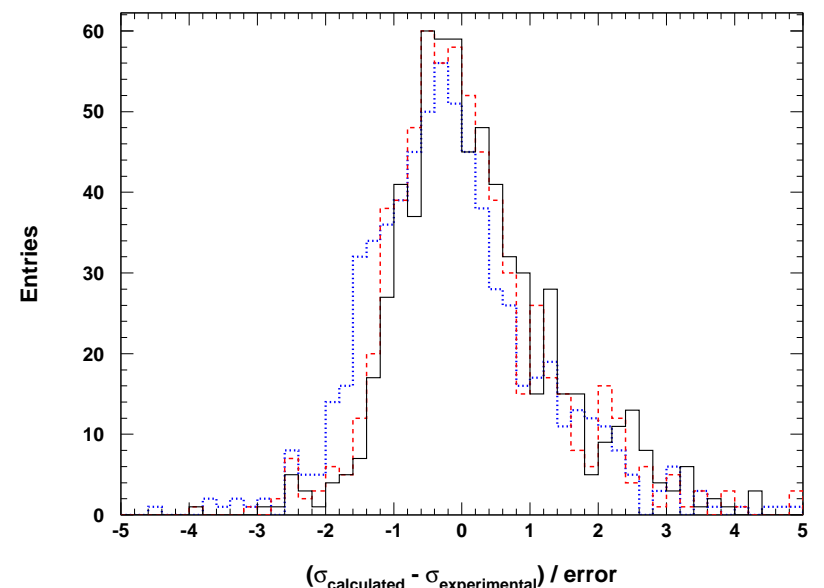

Fig. 13. Difference between $L_{3}$ subshell cross sections calculated by the three generators and experimental data, divided by the related experimental uncertainties: ISICS 2011 in ECPSSR-UA configuration (solid black histogram), ERCS08 (red dashed histogram) and LIO (dotted blue histogram) in default configuration.

ERCS08 default configurations fail. In this respect, however, it is worthwhile to note that in the case of tungsten, as well as for other test cases concerning heavy elements and relatively high ( $\gtrsim 5-7 \mathrm{MeV}$ ) energy, the Hartree-Slater correction does not improve the accuracy of ECPSSR calculations, actually the opposite.

TABLE III

CONTINGENCY TABLE TO ESTIMATE THE EQUIVALENT ACCURACY OF ECPSSR K SHELL CROSS SECTIONS USING EADL AND BEARDEN AND BURR'S BINDING ENERGIES

\begin{tabular}{|l|c|c|}
\hline$\chi^{2}$ test outcome & ISICS 2011 & ERCS08 \\
\hline Pass & 51 & 51 \\
Fail & 15 & 15 \\
\hline p-value Fisher test & \multicolumn{2}{|c|}{1} \\
p-value Pearson $\chi^{2}$ & \multicolumn{2}{|c|}{1} \\
p-value Yates $\chi^{2}$ & \multicolumn{2}{|c|}{0.835} \\
\hline$\chi^{2}$ test outcome & ISICS 2011 & KIO \\
\hline Pass & 51 & 47 \\
Fail & 15 & 19 \\
\hline p-value Fisher test & \multicolumn{2}{|c|}{0.551} \\
p-value Pearson $\chi^{2}$ & \multicolumn{2}{|c|}{0.426} \\
p-value Yates $\chi^{2}$ & \multicolumn{2}{|c|}{0.550} \\
\hline
\end{tabular}

\section{B. L shell}

The p-values of the $\chi^{2}$ test comparing calculated and experimental $L_{1}, L_{2}$ and $L_{3}$ subshell cross sections are reported for each tested element in Tables IV VI Whenever the data for a given element were present in both experimental compilations [37], [38], only the results associated with the compilation exhibiting the larger p-values are listed in the tables. The results are listed for ERCS08 and LIO in their default configuration, for ISICS 2011 in the ECPSRR-UA and plain ECPSSR configurations, which are similar to the default configurations of the other codes.

The results are summarized in Table VII where the entries for "pass", "fail" and efficiency correspond to the same
TABLE IV

P-VALUES OF THE $\chi^{2}$ TEST COMPARING CALCULATED AND EXPERIMENTAL $L_{1}$ SUBSHELL IONIZATION CROSS SECTIONS BY PROTON IMPACT

\begin{tabular}{|c|c|c|c|c|}
\hline \multirow[b]{2}{*}{ Z } & \multicolumn{2}{|c|}{ ISICS 2011} & ERCS08 & LIO \\
\hline & ECPSSR & ECPSSR-UA & Default & Default \\
\hline 45 & 0.030 & 0.004 & 0.021 & 0.079 \\
\hline 46 & 0.598 & 0.528 & 0.678 & 0.686 \\
\hline 47 & 0.966 & 0.951 & 0.971 & 0.979 \\
\hline 48 & 0.850 & 0.858 & 0.865 & 0.862 \\
\hline 49 & 0.067 & 0.087 & 0.056 & 0.056 \\
\hline 50 & 0.197 & 0.122 & 0.275 & 0.344 \\
\hline 51 & 0.946 & 0.972 & 0.936 & 0.910 \\
\hline 52 & 0.474 & 0.419 & 0.563 & 0.533 \\
\hline 59 & 0.056 & 0.101 & 0.040 & 0.022 \\
\hline 62 & 0.981 & 0.977 & 0.992 & 0.988 \\
\hline 64 & 0.263 & 0.243 & 0.236 & 0.212 \\
\hline 65 & 0.974 & 0.990 & 0.924 & 0.865 \\
\hline 66 & 0.951 & 0.953 & 0.939 & 0.938 \\
\hline 67 & 0.126 & 0.127 & 0.085 & 0.084 \\
\hline 70 & 0.619 & 0.619 & 0.553 & 0.552 \\
\hline 72 & 0.333 & 0.333 & 0.418 & 0.389 \\
\hline 73 & 0.929 & 0.929 & 0.928 & 0.911 \\
\hline 74 & $<0.001$ & $<0.001$ & $<0.001$ & $<0.001$ \\
\hline 75 & 0.975 & 0.975 & 0.986 & 0.986 \\
\hline 76 & 0.020 & 0.020 & 0.003 & 0.003 \\
\hline 77 & $<0.001$ & $<0.001$ & $<0.001$ & $<0.001$ \\
\hline 78 & $<0.001$ & $<0.001$ & $<0.001$ & $<0.001$ \\
\hline 79 & 0.916 & 0.916 & 0.873 & 0.877 \\
\hline 81 & $<0.001$ & $<0.001$ & $<0.001$ & $<0.001$ \\
\hline 82 & $<0.001$ & $<0.001$ & $<0.001$ & $<0.001$ \\
\hline 83 & 0.124 & 0.124 & 0.076 & 0.082 \\
\hline 90 & 0.003 & 0.003 & 0.006 & 0.007 \\
\hline 92 & $<0.001$ & $<0.001$ & $<0.001$ & $<0.001$ \\
\hline
\end{tabular}

definitions as in the previous section. ISICS 2011 ECPSSR$\mathrm{UA}$ is the most efficient at reproducing experimental cross sections, nevertheless, as shown in the contingency tables in Table VIII, the differences in efficiency are not statistically significant at $95 \%$ confidence level.

\section{Effect of atomic binding energies}

A detailed study [47] devoted to various atomic electron binding energy compilations in the context of Monte Carlo particle transport highlighted the effect of these atomic parameters in the calculation of proton ionization cross sections, regarding its accuracy with respect to experimental data. The effects are especially visible in the resulting values of $\mathrm{K}$ shell cross sections.

As commented in section II-B ERCS08 includes an ad hoc collection of binding energies, that does not correspond to any of the compilations used by major Monte Carlo systems and specialized PIXE codes. A test was performed to estimate the impact of this collection on the accuracy of the cross sections by running ISICS with ERCS08 binding energies: a series of $\chi^{2}$ test compared the compatibility of plain ECPSSR $\mathrm{K}$ shell cross sections calculated by ISICS based on either its default (Bearden and Burr [29]) or ERCS08 binding energies with experimental data at 0.05 significance level. The efficiency at reproducing experimental data raised from $0.67 \pm 0.06$ with Bearden and Burr binding energies to $0.80 \pm 0.05$ with ERCS08 binding energies.

More extensive documentation of the sources of ERCS08 binding energies would be beneficial to optimize the accuracy 
TABLE I

P-VALUES OF THE $\chi^{2}$ TEST COMPARING CALCULATED AND EXPERIMENTAL K SHELL IONIZATION CROSS SECTIONS BY PROTON IMPACT

\begin{tabular}{|c|c|c|c|c|c|c|c|}
\hline \multirow[b]{2}{*}{$\mathrm{Z}$} & \multicolumn{5}{|c|}{ ISICS 2011} & ERCS08 & KIO \\
\hline & ECPSSR & ECPSSR-HS & ECPSSR-UA & ECPSSR-HE & ECPSSR-HS-UA & Default & Default \\
\hline 6 & 0.751 & 0.944 & 0.751 & 0.774 & 0.944 & 0.985 & 0.860 \\
\hline 7 & $<0.001$ & 0.079 & $<0.001$ & $<0.001$ & 0.079 & 0.885 & $<0.001$ \\
\hline 8 & 0.986 & 0.986 & 0.983 & 0.986 & 0.987 & 0.945 & 0.986 \\
\hline 9 & 0.800 & 0.733 & 0.800 & 0.784 & 0.733 & 0.943 & 0.809 \\
\hline 10 & 0.999 & 0.996 & 0.999 & 0.999 & 0.996 & 0.995 & 1.000 \\
\hline 12 & 0.039 & 0.551 & 0.012 & 0.040 & 0.344 & 0.725 & 0.057 \\
\hline 13 & 0.003 & 0.079 & 0.001 & $<0.001$ & 0.061 & 0.292 & 0.004 \\
\hline 14 & 0.014 & 0.029 & 0.014 & 0.013 & 0.029 & 0.096 & 0.016 \\
\hline 15 & 0.710 & 0.612 & 0.710 & 0.706 & 0.612 & 0.515 & 0.695 \\
\hline 16 & 0.802 & 0.719 & 0.802 & 0.801 & 0.719 & 0.632 & 0.790 \\
\hline 17 & 0.650 & 0.732 & 0.650 & 0.657 & 0.732 & 0.787 & 0.666 \\
\hline 18 & 0.403 & 0.815 & 0.599 & 0.434 & 0.945 & 0.938 & 0.448 \\
\hline 19 & 0.993 & 0.989 & 0.993 & 0.993 & 0.989 & 0.985 & 0.993 \\
\hline 20 & 0.029 & 0.117 & 0.029 & 0.020 & 0.117 & 0.178 & 0.036 \\
\hline 21 & 0.257 & 1.000 & 0.257 & 0.331 & 1.000 & 1.000 & 0.490 \\
\hline 22 & 0.126 & 0.118 & 0.123 & 0.142 & 0.128 & 0.046 & 0.154 \\
\hline 23 & 0.059 & 0.517 & 0.053 & 0.082 & 0.499 & 0.537 & 0.111 \\
\hline 24 & 0.374 & 0.848 & 0.344 & 0.448 & 0.830 & 0.832 & 0.511 \\
\hline 25 & 0.405 & 0.526 & 0.276 & 0.417 & 0.387 & 0.373 & 0.508 \\
\hline 26 & 0.987 & 0.739 & 0.947 & 0.987 & 0.572 & 0.365 & 0.988 \\
\hline 27 & 0.969 & 0.983 & 0.965 & 0.980 & 0.980 & 0.936 & 0.982 \\
\hline 28 & 0.177 & 0.491 & 0.096 & 0.158 & 0.204 & 0.314 & 0.225 \\
\hline 29 & 0.653 & 0.890 & 0.504 & 0.749 & 0.811 & 0.806 & 0.812 \\
\hline 30 & 0.997 & 0.990 & 0.994 & 0.997 & 0.984 & 0.971 & 0.999 \\
\hline 31 & 0.042 & 0.381 & 0.042 & 0.075 & 0.381 & 0.465 & 0.083 \\
\hline 32 & 0.001 & 0.138 & 0.001 & 0.004 & 0.120 & 0.208 & 0.006 \\
\hline 33 & 0.066 & 0.392 & 0.066 & 0.104 & 0.392 & 0.496 & 0.143 \\
\hline 34 & 0.409 & 0.629 & 0.411 & 0.482 & 0.639 & 0.641 & 0.464 \\
\hline 35 & 0.248 & 0.312 & 0.248 & 0.291 & 0.312 & 0.297 & 0.249 \\
\hline 36 & 0.235 & 0.522 & 0.235 & 0.320 & 0.522 & 0.538 & 0.383 \\
\hline 37 & 0.105 & 0.147 & 0.105 & 0.119 & 0.147 & 0.143 & 0.087 \\
\hline 38 & 0.019 & 0.023 & 0.019 & 0.025 & 0.023 & 0.031 & 0.049 \\
\hline 39 & 0.109 & 0.189 & 0.109 & 0.121 & 0.189 & 0.205 & 0.147 \\
\hline 40 & 0.039 & 0.067 & 0.037 & 0.056 & 0.065 & 0.080 & 0.088 \\
\hline 41 & 0.001 & 0.002 & 0.002 & 0.003 & 0.003 & 0.003 & 0.002 \\
\hline 42 & 0.998 & 0.999 & 0.998 & 1.000 & 0.999 & 0.999 & 0.999 \\
\hline 45 & $<0.001$ & $<0.001$ & $<0.001$ & $<0.001$ & $<0.001$ & $<0.001$ & $<0.001$ \\
\hline 46 & 0.621 & 0.981 & 0.573 & 0.614 & 0.936 & 0.957 & 0.851 \\
\hline 47 & 0.332 & 0.379 & 0.332 & 0.562 & 0.398 & 0.378 & 0.589 \\
\hline 48 & 0.001 & $<0.001$ & 0.001 & 0.007 & $<0.001$ & $<0.001$ & 0.004 \\
\hline 49 & $<0.001$ & $<0.001$ & $<0.001$ & $<0.001$ & $<0.001$ & $<0.001$ & $<0.001$ \\
\hline 50 & 0.946 & 0.957 & 0.946 & 0.942 & 0.957 & 0.962 & 0.980 \\
\hline 51 & 0.787 & 0.874 & 0.787 & 0.812 & 0.874 & 0.889 & 0.961 \\
\hline 52 & $<0.001$ & $<0.001$ & $<0.001$ & $<0.001$ & $<0.001$ & $<0.001$ & $<0.001$ \\
\hline 53 & 0.057 & 0.014 & 0.057 & 0.119 & 0.014 & 0.018 & 0.305 \\
\hline 55 & 0.327 & 0.468 & 0.327 & 0.247 & 0.468 & 0.468 & 0.280 \\
\hline 56 & 0.310 & 0.922 & 0.310 & 0.147 & 0.922 & 0.914 & 0.248 \\
\hline 57 & 0.056 & 0.170 & 0.056 & 0.037 & 0.170 & 0.166 & 0.052 \\
\hline 58 & 0.073 & 0.579 & 0.073 & 0.063 & 0.579 & 0.588 & 0.378 \\
\hline 59 & 0.010 & 0.009 & 0.010 & 0.014 & 0.009 & 0.009 & 0.031 \\
\hline 60 & 0.243 & 0.596 & 0.243 & 0.148 & 0.596 & 0.609 & 0.355 \\
\hline 62 & 0.218 & 0.976 & 0.218 & 0.110 & 0.976 & 0.978 & 0.514 \\
\hline 63 & $<0.001$ & $<0.001$ & 0.001 & 0.002 & $<0.001$ & $<0.001$ & 0.010 \\
\hline 64 & 0.394 & 0.324 & 0.394 & 0.082 & 0.324 & 0.340 & 0.500 \\
\hline 65 & 0.008 & 0.522 & 0.008 & 0.003 & 0.522 & 0.527 & 0.025 \\
\hline 67 & 0.020 & 0.997 & 0.020 & 0.001 & 0.998 & 0.997 & 0.011 \\
\hline 69 & 0.014 & 0.014 & 0.014 & 0.022 & 0.007 & 0.015 & 0.049 \\
\hline 70 & $<0.001$ & $<0.001$ & $<0.001$ & 0.008 & $<0.001$ & $<0.001$ & $<0.001$ \\
\hline 72 & 0.275 & 0.647 & 0.275 & 0.311 & 0.647 & 0.650 & 0.645 \\
\hline 73 & 0.029 & 0.004 & 0.029 & 0.019 & 0.004 & 0.005 & 0.018 \\
\hline 74 & 0.232 & $<0.001$ & 0.232 & 0.350 & $<0.001$ & $<0.001$ & 0.719 \\
\hline 75 & 0.075 & 0.070 & 0.075 & 0.093 & 0.070 & 0.072 & 0.185 \\
\hline 78 & 0.186 & 0.528 & 0.186 & 0.187 & 0.092 & 0.094 & 0.001 \\
\hline 79 & 0.107 & $<0.001$ & 0.107 & 0.454 & $<0.001$ & $<0.001$ & 0.667 \\
\hline 82 & 0.003 & $<0.001$ & 0.003 & 0.128 & $<0.001$ & $<0.001$ & 0.022 \\
\hline 83 & 0.001 & 0.256 & 0.001 & 0.001 & 0.256 & 0.265 & 0.052 \\
\hline 90 & 0.007 & $<0.001$ & 0.007 & 0.003 & $<0.001$ & $<0.001$ & 0.001 \\
\hline 92 & 0.136 & 0.646 & 0.136 & 0.158 & 0.646 & 0.657 & 0.524 \\
\hline
\end{tabular}


TABLE II

SUMMARY OF THE $\chi^{2}$ TEST RESULTS OF K SHELL IONIZATION CROSS SECTIONS BY PROTON IMPACT

\begin{tabular}{|l|ccccc|c|c|}
\hline & & & \multicolumn{3}{c|}{ ISICS 2011 } & KRCS08 & KIO \\
& ECPSSR & ECPSSR-HS & ECPSSR-UA & ECPSSR-HE & ECPSSR-HS-UA & $\begin{array}{c}\text { Default } \\
\text { Default }\end{array}$ \\
\hline Tested elements & 66 & 66 & 66 & 66 & 66 & 66 & 66 \\
Pass & 44 & 51 & 44 & 46 & 51 & 51 & 47 \\
Fail & 22 & 15 & 22 & 20 & 15 & 15 & 19 \\
Efficiency & $0.67 \pm 0.06$ & $0.77 \pm 0.05$ & $0.67 \pm 0.06$ & $0.70 \pm 0.06$ & $0.77 \pm 0.05$ & $0.77 \pm 0.05$ & $0.71 \pm 0.06$ \\
\hline
\end{tabular}

TABLE VII

SUMMARY OF $\chi^{2}$ TEST RESULTS OF L SUBSHELL IONIZATION CROSS SECTIONS BY PROTON IMPACT

\begin{tabular}{|cl|cc|c|c|}
\hline & & \multicolumn{2}{|c|}{ ISICS 2011 } & ERCS08 & LIO \\
& & ECPSSR & ECPSSR-UA & Default & Default \\
\hline \multirow{4}{*}{$L_{1}$} & Elements & 28 & 28 & 28 & 28 \\
& Pass & 19 & 19 & 18 & 19 \\
& Fail & 9 & 9 & 10 & 9 \\
& Efficiency & $0.53 \pm 0.09$ & $0.53 \pm 0.09$ & $0.48 \pm 0.09$ & $0.50 \pm 0.09$ \\
\hline \multirow{4}{*}{$L_{2}$} & Elements & 28 & 28 & 28 & 28 \\
& Pass & 19 & 22 & 20 & 18 \\
& Fail & 9 & 6 & 8 & 10 \\
& Efficiency & $0.68 \pm 0.09$ & $0.79 \pm 0.08$ & $0.71 \pm 0.09$ & $0.64 \pm 0.09$ \\
\hline \multirow{4}{*}{$L_{3}$} & Elements & 28 & 28 & 28 & 28 \\
& Pass & 25 & 25 & 26 & 21 \\
& Fail & 3 & 3 & 2 & 7 \\
& Efficiency & $0.89 \pm 0.06$ & $0.89 \pm 0.06$ & $0.93 \pm 0.05$ & $0.75 \pm 0.08$ \\
\hline \multirow{4}{*}{$L$} & Elements & 84 & 84 & 84 & 84 \\
& Pass & 63 & 66 & 64 & 58 \\
& Fail & 21 & 18 & 20 & 26 \\
& Efficiency & $0.75 \pm 0.05$ & $0.79 \pm 0.04$ & $0.76 \pm 0.59$ & $0.69 \pm 0.05$ \\
\hline
\end{tabular}

TABLE V

P-VALUES OF THE $\chi^{2}$ TEST COMPARING CALCULATED AND EXPERIMENTAL $L_{2}$ SUBSHELL IONIZATION CROSS SECTIONS BY PROTON IMPACT

\begin{tabular}{|l|cc|c|c|}
\hline $\mathrm{Z}$ & \multicolumn{2}{|c|}{ ISICS 2011 } & ERCS08 & LIO \\
& ECPSSR & ECPSSR-UA & Default & Default \\
\hline 45 & 0.001 & 0.057 & 0.023 & $<0.001$ \\
46 & $<0.001$ & 0.008 & 0.002 & $<0.001$ \\
47 & 0.015 & 0.139 & 0.067 & 0.029 \\
49 & 0.101 & 0.121 & 0.080 & 0.084 \\
50 & 0.082 & 0.256 & 0.136 & 0.055 \\
51 & 0.092 & 0.163 & 0.132 & 0.073 \\
52 & 1.000 & 1.000 & 1.000 & 1.000 \\
59 & 0.718 & 0.722 & 0.822 & 0.761 \\
62 & 0.350 & 0.654 & 0.581 & 0.258 \\
64 & 0.005 & 0.060 & 0.026 & 0.002 \\
65 & $<0.001$ & $<0.001$ & $<0.001$ & $<0.001$ \\
66 & 0.001 & $<0.001$ & $<0.001$ & $<0.001$ \\
67 & 0.173 & 0.862 & 0.821 & 0.777 \\
70 & 0.129 & 0.312 & 0.196 & 0.093 \\
72 & 0.984 & 0.991 & 0.065 & 0.064 \\
73 & 0.054 & 0.116 & 0.967 & 0.962 \\
74 & 0.341 & 0.453 & 0.231 & 0.028 \\
75 & 0.398 & 0.456 & 0.294 & 0.185 \\
76 & 0.762 & 0.871 & 0.677 & 0.556 \\
77 & 0.331 & 0.433 & 0.208 & 0.166 \\
78 & 0.655 & 0.713 & 0.500 & 0.478 \\
79 & 1.000 & 1.000 & 0.998 & 0.999 \\
81 & 0.018 & 0.018 & 0.009 & 0.010 \\
82 & 0.841 & 0.843 & 0.711 & 0.728 \\
83 & 0.322 & 0.322 & 0.187 & 0.187 \\
90 & 0.049 & 0.049 & 0.023 & 0.025 \\
92 & $<0.001$ & $<0.001$ & $<0.001$ & $<0.001$ \\
\hline
\end{tabular}

TABLE VI

P-VALUES OF THE $\chi^{2}$ TEST COMPARING CALCULATED AND EXPERIMENTAL $L_{3}$ SUBSHELL IONIZATION CROSS SECTIONS BY PROTON IMPACT

\begin{tabular}{|c|cc|c|c|}
\hline $\mathrm{Z}$ & \multicolumn{2}{|c|}{ ISICS 2011 } & ERCS08 & LIO \\
& ECPSSR & ECPSSR-UA & Default & Default \\
\hline 45 & 0.235 & 0.987 & 0.948 & 0.168 \\
46 & 0.061 & 0.829 & 0.620 & 0.038 \\
47 & 0.436 & 0.969 & 0.888 & 0.347 \\
48 & 0.909 & 0.949 & 0.919 & 0.891 \\
49 & 0.679 & 0.978 & 0.907 & 0.583 \\
50 & 0.221 & 0.137 & 0.172 & 0.204 \\
51 & 0.999 & 0.999 & 1.000 & 0.999 \\
52 & 0.535 & 0.463 & 0.608 & 0.594 \\
59 & 0.019 & 0.399 & 0.245 & 0.010 \\
62 & 0.087 & 0.764 & 0.586 & 0.044 \\
64 & 0.783 & 0.274 & 0.581 & 0.679 \\
65 & 0.309 & 0.912 & 0.833 & 0.188 \\
66 & 0.643 & 0.502 & 0.589 & 0.592 \\
67 & 0.102 & 0.512 & 0.402 & 0.048 \\
70 & 0.599 & 0.991 & 0.963 & 0.394 \\
72 & 0.970 & 0.950 & 0.992 & 0.984 \\
73 & 0.525 & 0.867 & 0.652 & 0.339 \\
74 & 0.701 & 0.928 & 0.807 & 0.549 \\
75 & 0.960 & 0.960 & 0.992 & 0.971 \\
76 & $<0.001$ & $<0.001$ & 0.004 & $<0.001$ \\
77 & 1.000 & 0.979 & 0.999 & 1.000 \\
78 & $<0.001$ & $<0.001$ & $<0.001$ & $<0.001$ \\
79 & 1.000 & 1.000 & 1.000 & 1.000 \\
81 & 0.060 & 0.109 & 0.066 & 0.038 \\
82 & 1.000 & 1.000 & 1.000 & 1.000 \\
83 & 0.460 & 0.508 & 0.560 & 0.430 \\
90 & 0.963 & 0.994 & 0.978 & 0.907 \\
92 & 0.085 & 0.021 & 0.080 & 0.179 \\
\hline
\end{tabular}


TABLE VIII

CONTINGENCY TABLE TO ESTIMATE THE EQUIVALENT ACCURACY OF ECPSSR L SHELL CROSS SECTIONS USING EADL AND BEARDEN AND BURR'S BINDING ENERGIES

\begin{tabular}{|l|c|c|}
\hline$\chi^{2}$ test outcome & ISICS 2011 & ERCS08 \\
\hline Pass & 66 & 64 \\
Fail & 18 & 20 \\
\hline p-value Fisher test & \multicolumn{2}{|c|}{0.854} \\
p-value Pearson $\chi^{2}$ & \multicolumn{2}{|c|}{0.712} \\
p-value Yates $\chi^{2}$ & \multicolumn{2}{|c|}{0.854} \\
\hline$\chi^{2}$ test outcome & ISICS 2011 & LIO \\
\hline Pass & 66 & 58 \\
Fail & 18 & 26 \\
\hline p-value Fisher test & \multicolumn{2}{|c|}{0.161} \\
p-value Pearson $\chi^{2}$ & \multicolumn{2}{|c|}{0.219} \\
p-value Yates $\chi^{2}$ & 0.160 \\
\hline
\end{tabular}

of ECPSSR cross section calculations.

\section{Empirical scaling}

Although successful at describing ionization cross sections over a wide energy range relevant to PIXE experimental applications, the ECPSSR theory fails at reproducing experimental measurements in some conditions, for instance at low energies (below approximately $1 \mathrm{MeV}$ ). The HartreeSlater correction applied to the calculation $\mathrm{K}$ shell cross sections in part overcomes these deficiencies; nevertheless, as previously pointed out, this modeling approach exhibits other shortcomings, namely for heavy elements at higher energies (approximately above a few MeV).

An empirical correction has been developed by Paul and Muhr [48] as a scaling function $s_{c}^{\prime}$ to be applied to ECPSSR cross sections:

$$
\sigma_{\text {scaled }}=s_{c}^{\prime} \cdot \sigma_{\mathrm{ECPSSR}}
$$

The parameters of the scaling function have been fitted to experimental data.

Tabulations of empirically scaled cross sections for $\mathrm{K}$ shell have been published by Paul and Sacher [36]; the procedure of their calculation was modified with respect to that adopted in [48], but the updated scaling function is not documented in [36]. These tabulations are included in the PIXE data library [19].

Empirically scaled $\mathrm{K}$ shell cross sections are calculated by the KIOKC executable in Šmit's software system; this program uses the atomic binding energies of the 1978 edition of the Table of Isotopes, consistently with those used in [48].

An implementation of Paul and Muhr's scaling function has been developed for use with Geant 4 and is intended for release in a forthcoming Geant 4 version. Using the electron binding energies of the 1978 Table of Isotopes, it has been verified to reproduce the values reported in [48] for a selection of elements and energies. This scaling function can be applied to ECPSSR cross sections calculated by other generators to improve the calculation accuracy as an alternative to other theoretical variants, like the Hartree-Slater and United Atom corrections.

The use of this scaling function could be source of systematic effects. The parameters defining the empirical scaling

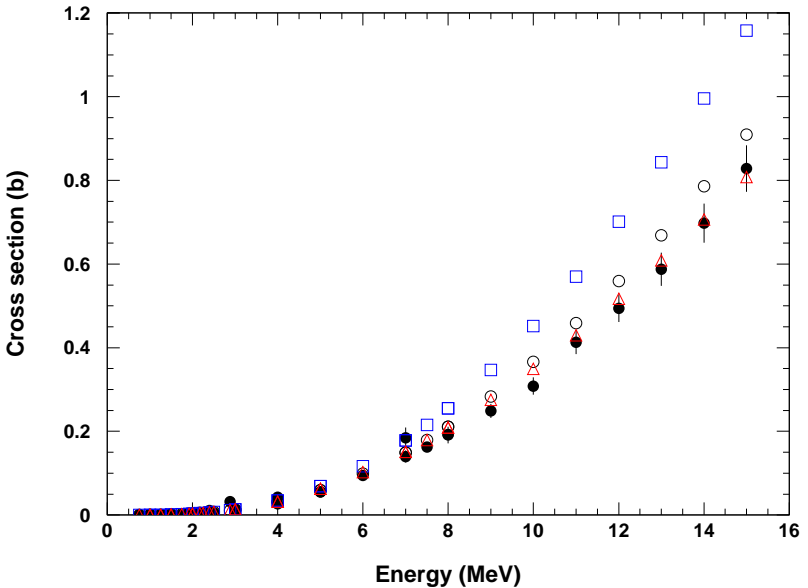

Fig. 14. $\mathrm{K}$ shell ionization cross sections by proton impact on lead: experimental data from [36] (black filled circles), theoretical calculations by ISICS 2011 configured with the ECPSSR-HS-UA (empty blues squares) and plain ECPSSR (empty black circles) options, and ECPSSR values scaled by the empirical scaling function defined in [48] (empty red triangles).

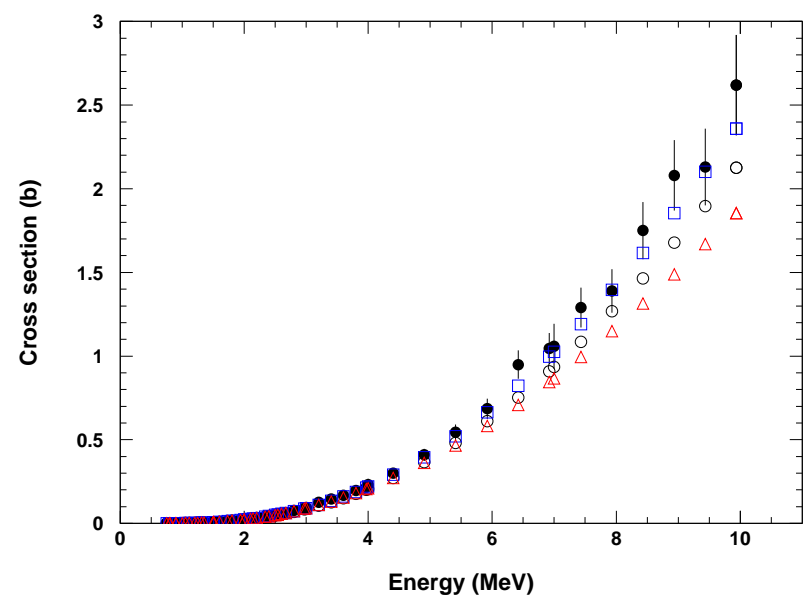

Fig. 15. K shell ionization cross sections by proton impact on holmium: experimental data from [36] (black filled circles), theoretical calculations by ISICS 2011 configured with the ECPSSR-HS-UA (empty blue squares) and plain ECPSSR (empty black circles) options, and ECPSSR values scaled by the empirical scaling function defined in [48] (empty red triangles).

function documented in [48] derive from a fit to the experimental data involving calculations of ECPSSR cross sections; therefore, strictly speaking, that empirical correction should be applied only to identically calculated ECPSSR cross sections. The values calculated by the three generators analyzed in this paper slightly differ from the ECPSSR cross sections reported in [48] for selected elements and energies; however, since the discrepancies are relatively small (e.g. less than $1 \%$ for ECPSSR cross cross sections calculated by ISICS), the systematic error due to the application of the empirical scaling functions to other ECPSSR calculations than those used in [48] would also be small, and negligible with respect to the experimental uncertainties of $\mathrm{K}$ shell cross sections.

The contribution of the empirical scaling function to the cross section accuracy has been estimated according to the 
TABLE IX

EFFICIENCY OF EMPIRICALLY SCALED AND THEORETICAL K SHELL IONIZATION CROSS SECTION CALCULATIONS

\begin{tabular}{|l|ccc|cc|}
\hline & \multicolumn{3}{|c|}{ ISICS 2011 } & \multicolumn{2}{c|}{ Śmit } \\
& ECPSSR & ECPSSR-HS-UA & Scaled ECPSSR & KIO & KIOKC \\
\hline Tested elements & 66 & 66 & 66 & 66 & 66 \\
Pass & 44 & 51 & 54 & 47 & 54 \\
Fail & 22 & 15 & 12 & 19 & 12 \\
Efficiency & $0.67 \pm 0.06$ & $0.77 \pm 0.05$ & $0.82 \pm 0.05$ & $0.71 \pm 0.06$ & $0.82 \pm 0.05$ \\
\hline
\end{tabular}

procedure described in section IV-C, this process does not constitute a validation, since the experimental data to which the calculated cross sections are compared are in large part the same used for fitting the parameters of the scaling function itself. The usual procedure to account for fitting constraints in a $\chi^{2}$ test, consisting of reducing the number of degrees of freedom accordingly, cannot be applied to this case due to incomplete knowledge of the fit configuration. In fact, the fits described in [48] concern groups of several elements, while in this analysis the $\chi^{2}$ tests are performed for individual elements; moreover, the experimental samples of Paul and Sacher's paper [36], used in this analysis, do not exactly coincide with the data on which the scaling function drawn from Paul and Muhr's paper [48] has been fitted, although a large fraction are common. The ECPSSR cross sections, which are involved in the fit, are slightly different in [48] and in this analysis. Therefore the results reported in the following should be considered only as a demonstration of the capability of the empirical scaling to describe the data.

The efficiency, defined as in the previous sections, is listed in Table IX for ECPSSR cross sections calculated by KIOKC and by ISICS scaled by Paul and Muhr's empirical function along with the efficiency of the ISICS ECPSSR and ECPSSRHS-UA options, and of KIO. The gain in efficiency due to the scaling function appears substantial, although, as illustrated in the contingency table reported in Table $\mathrm{X}$ the outcome of the statistical analysis is controversial: the hypothesis of equivalence of theoretical and empirically scaled ECPSSR cross sections is rejected with 0.05 significance by Pearson's $\chi^{2}$ test, but only with 0.1 significance by Yates' $\chi^{2}$ test and Fisher's test. The gain with respect to the theoretical ECPSSRHS-UA option is not statistically significant; nevertheless, in some experimental scenarios for which the theory does not reproduce the measurements accurately, for instance as illustrated in Fig. 14 the capability of applying empirical scaling corrections to the theoretical cross sections could be a valuable option. However, within the data sample analyzed in this paper, one can also identify cases where theoretical cross sections calculated with the Hartree-Slater and United Atom corrections are more accurate than empirically scaled ECPSSR ones; an example is shown in Fig. 15.

\section{E. Interpolated cross sections}

In the data driven approach to PIXE simulation described in [14], the cross sections calculated by analytical generators are tabulated at predefined energies and used in Monte Carlo simulation to compute cross sections corresponding to the energy of a given particle being tracked through matter.
TABLE X

CONTINGENCY TABLE TO ESTIMATE THE EQUIVALENT ACCURACY OF PLAIN AND EMPIRICALLY SCALED ISICS 2011 ECPSSR K SHELL CROSS SECTIONS

\begin{tabular}{|l|c|c|}
\hline$\chi^{2}$ test outcome & ECPSSR & Scaled \\
\hline Pass & 44 & 54 \\
Fail & 22 & 12 \\
\hline p-value Fisher test & \multicolumn{2}{|c|}{0.072} \\
p-value Pearson $\chi^{2}$ & \multicolumn{2}{|c|}{0.047} \\
p-value Yates $\chi^{2}$ & \multicolumn{2}{|c|}{0.073} \\
\hline
\end{tabular}

A test was performed to verify if the interpolation process performed in Geant 4 would significantly affect the accuracy of the theoretical cross sections.

The distribution of the relative difference between interpolated and theoretical ECPSSR cross sections is shown in Fig. 16 for $\mathrm{K}$ shell cross sections; the distributions for $\mathrm{L}$ subshells are similar. The data span the energy range covered by experimental measurements; the theoretical cross sections are generated by ISICS, while the interpolated values are computed by logarithmic interpolation of the tabulations produced by ISICS. The plot includes only truly interpolated values: cross sections calculated by the interpolation algorithm at energies corresponding to the tabulated values are excluded, since the differences are obviously null. The distribution can be described by a Gaussian with $0.17 \%$ mean and $0.11 \%$ standard deviation; the tail corresponds to regions of relatively steep variation of the cross sections as a function of energy, mostly occurring with light targets at low energies (below $1 \mathrm{MeV}$ ). The error due to interpolation is negligible with respect to the experimental uncertainties of the cross sections, as can be observed in Fig. 17

Interpolated cross sections exhibit the same compatibility with experiment as theoretically calculated ones: the outcome of the $\chi^{2}$ test, as rejection or non-rejection of the null hypothesis at 0.05 significance level, is strictly identical for ECPSSR cross sections calculated by ISICS and interpolated from tabulations produced with ISICS, when they are compared to the same experimental samples applying the same analysis criteria, although the test statistic assumes slightly different numerical values for the two categories in the individual test cases. This result holds for cross sections interpolated from ISICS 2008 tabulations with respect to theoretical values directly calculated by either ISICS 2008 or 2011.

From this test one evinces that the interpolation process used in Geant4 PIXE simulation [14] introduces a negligible perturbation to the accuracy of the cross sections calculated in the course of the simulation; it also confirms that the updates implemented in ISICS 2011 do not modify the accuracy 


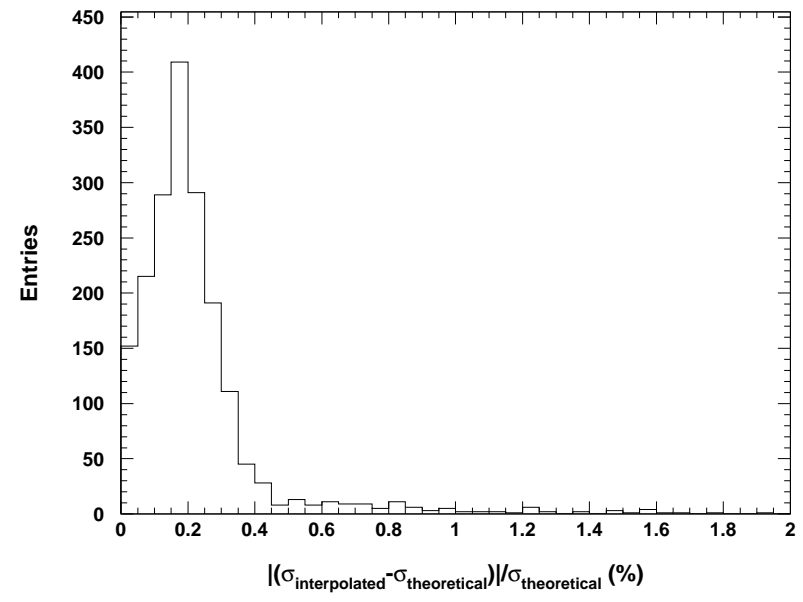

Fig. 16. Absolute relative difference between interpolated and theoretical ECPSSR $\mathrm{K}$ shell ionization cross sections by proton; the theoretical cross sections are produced by ISICS, while the interpolated values are calculated by the logarithmic interpolation algorithm encompassed in the data management component described in [14], based on tabulations produced by ISICS.

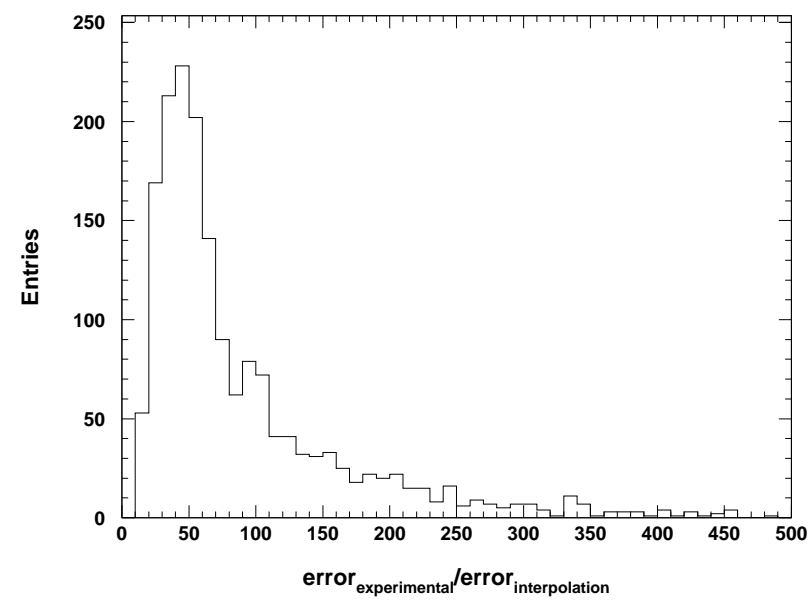

Fig. 17. Ratio of $\mathrm{K}$ shell cross section experimental uncertainties and interpolation errors; the interpolation error is defined as the absolute difference between interpolated and theoretical cross sections. The theoretical cross sections are produced by ISICS, while the interpolated values are calculated by the logarithmic interpolation algorithm encompassed in the data management component described in [14], based on tabulations produced by ISICS; the experimental uncertainties are taken from [36].

of Geant4 PIXE simulation with respect to the data library distributed with Geant4 9.4, which is based on the previous ISICS version.

It is worthwhile to stress that these results concerning the interpolation of cross section tabulations produced by ISICS concern the data library and interpolation algorithm described in [14]; they would not necessarily hold for tabulations adopting different sampling intervals or exploiting other interpolation algorithms.

\section{CONCLUSION}

Three publicly available theoretical generators of inner shell ionization by proton impact have been analyzed to assess the state-of-the-art in the field: the 2011 version of ISICS, the new code ERCS08 and Šmit's software system (here called KIO-LIO).

The accuracy achieved by the three generators has been estimated through the statistical comparison of their results with experimental $\mathrm{K}$ and $\mathrm{L}$ shell cross sections. The analysis confirms quantitatively the qualitative similarity of the three generators. For K shell ionization, ISICS and ERCS08 exhibit the same overall efficiency at reproducing experimental references, while the KIO system is slightly less accurate. For the L shell, ISICS achieves the highest efficiency and LIO the lowest: nevertheless, the differences among the generators are not statistically significant at 0.05 level. One can conclude that ISICS represents the state-of-the-art among freely available ECPSSR generators.

Although quite similar in their overall statistical performance, the three generators exhibit distinctive features for a few specific elements (for instance, for silicon $\mathrm{K}$ shell ionization).

Their documentation in the detailed results of this paper provides guidance to optimize the selection of cross section options in applications, which may be sensitive to the accuracy of the results for specific targets of experimental interest.

These results are relevant to the production of cross section tabulations for Monte Carlo particle transport, and the selection of optimal cross section options in simulation applications.

The analysis also assessed the equivalent compatibility with measurements of the 2011 and 2008 versions of ISICS; the latter was used for the production of the cross section data library released in Geant4 9.4 for PIXE simulation. Moreover, it demonstrated that the interpolation of tabulated values does not significantly affect the accuracy of the cross sections calculated in Geant4.

The PIXE data library has been extended to encompass tabulations produced by the other generators analyzed in this paper in addition to those based on ISICS, so that users can profit from the distinctive features of the three generators identified in this study. An updated version is intended to be released with a forthcoming version of the Geant4 toolkit and through the RSICC distribution center. An extension to Geant4 code is also foreseen to provide functionality for empirically scaling ECPSSR K shell cross sections.

Apart from the calculation of cross sections for the ionization of target atoms, which is the subject of this paper, the simulation of PIXE with Geant4 involves the atomic relaxation, whose accuracy is documented in [49]. Other issues concerning the consistent treatment of the discrete process of atomic relaxation along with the ionization process, which is affected by infrared divergences, are extensively discussed in [14].

\section{ACKNOWLEDGMENT}

The KIO-LIO code has been kindly provided to the authors by Žiga Šmit. The authors are grateful to Sam J. Cipolla, Vladimir Horvat and Žiga Šmit for extensive information on their codes and useful advice on how to use them, and to Barbara Setina Batič for valuable comments. The CERN 
Library, in particular Tullio Basaglia, has provided helpful assistance and essential reference material for this study.

\section{REFERENCES}

[1] W. Brandt and G. Lapicki, "Energy-loss effect in inner-shell Coulomb ionization by heavy charged particles", Phys. Rev. A, vol. 23, pp. 1717-1729, 1981.

[2] C. G. Ryan, D. R. Cousens, S. H. Sie, and W. L. Griffin, "Quantitative analysis of PIXE spectra in geoscience applications", Nucl. Instrum. Meth. B, vol. 49, no. 1-4, pp. 271-276, 1990.

[3] J. A. Maxwell, J. L. Campbell, and W. J. Teesdale, "The Guelph PIXE software package", Nucl. Instrum. Meth. B, vol. 43, no. 2, pp. 218-230, 1989.

[4] J. A. Maxwell, W. J. Teesdale, and J. L. Campbell, "The Guelph PIXE software package II", Nucl. Instrum. Meth. B, vol. 95, no. 3, pp. 407421, 1995.

[5] J. L. Campbell, T. L. Hopman, J. A. Maxwell, Z. Nejedly, "The Guelph PIXE software package III: Alternative proton database" Nucl. Instrum. Meth. B, vol. 170, pp. 193-204, 2000

[6] E. Clayton, "PIXAN, the Lucas Heights PIXE analysis computer package", Australian Nuclear Science and Technology Organisation, AAEC/M113, 1986.

[7] A. J. Antolak and G. S. Bench, "PIXEF: the Livermore PIXE spectrum analysis package, Nucl. Instrum. Meth. B, vol. 90, no. 1-4, pp. 596-601, 1994.

[8] G. Szabo and I. Borbely-Kiss, "PIXYKLM computer package for PIXE analyses" Nucl. Instrum. Meth. B, vol. 75, no. 1-4, pp. 123-126, 1993.

[9] K. Sera and S. Futatsugawa, "Personal computer aided data handling and analysis for PIXE", Nucl. Instrum. Meth. B, vol. 109-110, pp. 99104, 1996.

[10] I. Orlic, J. Makjanic, G. H. J. Tros and R. D. Vis, "TTPIXAN A package of computer programs for quantitative thick target PIXE analysis", Nucl. Instrum. Meth. B, vol. 49, no.1-4, pp. 166-172, 1990.

[11] K. K. Loh, C. H. Sow, I. Orlic and S. M. Tang, "Computer simulation of PIXE and $\mu$-PIXE spectra for inhomogeneous thick target analysis", Nucl. Instrum. Meth. B, vol. 77, no. 1-4, pp. 132-136, 1993.

[12] I. Orlic, S. Zhou, J. L. Sanchez, F. Watt, and S. M. Tang, "Virtual PIXE and RBS laboratory", Nucl. Instrum. Meth. B, vol. 150, no. 1-2, pp. 83-89, 1999.

[13] C. Pascual-Izarra, N.P. Barradas, and M. A. Reis, "LibCPIXE: A PIXE simulation open-source library for multilayered samples", Nucl. Instrum. Meth. B, vol. 249, pp. 820-822, 2006.

[14] M. G. Pia et al., "PIXE simulation with Geant4", IEEE Trans. Nucl. Sci., vol. 56, no. 6, pp. 3614 - 3649, 2009.

[15] S. Agostinelli et al., "Geant4 - a simulation toolkit" Nucl. Instrum. Meth. A, vol. 506, no. 3, pp. 250-303, 2003.

[16] J. Allison et al., "Geant4 Developments and Applications" IEEE Trans. Nucl. Sci., vol. 53, no. 1, pp. 270-278, 2006.

[17] E. Merzbacher and H. Lewis, Handbuch der Physik, vol. 34, pp. 166192, Springer, Berlin, 1958.

[18] Z. Liu and S. J. Cipolla, "ISICS: A program for calculating K-, Land M-shell cross sections from ECPSSR theory using a personal computer", Comp. Phys. Comm., vol. 97, no. 3, pp. 315-330, 1996.

[19] M. G. Pia et al., PIXE2010: Proton/alpha Ionisation (K, L, M shell), Tabulated Cross Section Library. Available from the Radiation Safety Information Computational Center (RSICC) at Oak Ridge National Laboratory as DLC-246.

[20] V. Horvat, "ERCS08: A FORTRAN program equipped with a Windows graphics user interface that calculates ECPSSR cross sections for the removal of atomic electrons", Comp. Phys. Comm., vol. 180, no.6, pp. 995-1003, 2009.

[21] Z. Šmit, "K-shell ECPSSR cross sections for analytical applications", Nucl. Instrum. Meth. B, vol. 36, no. 3, pp. 254-258, 1989.

[22] IEEE Computer Society, "IEEE Standard for Software Verification and Validation", IEEE Std 1012-2004, Jun. 2005.

[23] S. J. Cipolla, "The united atom approximation option in the ISICS program to calculate K-, L-, and M-shell cross sections from PWBA and ECPSSR theory", Nucl. Instrum. Meth. B, vol. 261, pp. 142-144, 2007

[24] G. Lapicki, "The status of theoretical K-shell ionization cross sections by protons", X-Ray Spectrom., vol. 34, pp. 269-278, 2005.

[25] G. Lapicki, "Scaling of analytical cross sections for K-shell ionization by nonrelativistic protons to cross sections by protons at relativistic velocities", J. Phys. B, vol. 41, pp. 115201 (13pp), 2008.
[26] S. J. Cipolla, "An improved version of ISICS: a program for calculating K-, L-, and M-shell cross sections from PWBA and ECPSSR theory using a personal computer", Comp. Phys. Comm., vol. 176, pp. 157159, 2007.

[27] S. J. Cipolla, "ISICS2008: An expanded version of ISICS for calculating K-, L-, and M-shell cross sections from PWBA and ECPSSR theory", Comp. Phys. Comm., vol. 180, no .9, pp. 1716-1717, 2009.

[28] S. J. Cipolla, "ISICS2011, an updated version of ISICS: A program for calculation K-, L-, and M-shell cross sections from PWBA and ECPSSR theories using a personal computer", Comp. Phys. Comm., vol. 182 , no. 11 , pp. 2439-2440, 2011.

[29] J. A. Bearden and A. F. Burr, "Reevaluation of X-Ray Atomic Energy Levels", Rev. Mod. Phys., vol. 39, pp. 125142, 1967.

[30] A. C. Thompson et al., "X-ray Data Booklet", 3rd ed., Lawrence Berkeley Natl. Lab., 2009.

[31] D. R. Lide ed., "CRC Handbook of Chemistry and Physics", 90th ed., Boca Raton, FL, 2009.

[32] R. D. Deslattes et al., "X-ray transition energies: new approach to a comprehensive evaluation", Rev. Mod. Phys., vol. 75, pp. 35-99, 2003.

[33] M. Lederer and V. S. Shirley eds., "Table of Isotopes, 7th ed.", John Wiley \& Sons, New York, 1978.

[34] S. T. Perkins et al., "Tables and Graphs of Atomic Subshell and Relaxation Data Derived from the LLNL Evaluated Atomic Data Library (EADL)", Z=1-100, UCRL-50400 Vol. 30, 1997.

[35] K. D. Sevier, "Atomic electron binding energies", Atom. Data Nucl. Data Tables, vol. 24, pp. 323-371, 1979.

[36] H. Paul and J. Sacher, "Fitted empirical reference cross sections for K-shell ionization by protons", At. Data Nucl. Data Tab., vol. 42, pp. 105-156, 1989.

[37] R. S. Sokhi and D. Crumpton, "Experimental L-Shell X-Ray Production and Ionization Cross Sections for Proton Impact", At. Data Nucl. Data Tables, vol. 30, pp. 49-124, 1984.

[38] I. Orlic, J. Sow, and S.M. Tang, "Experimental L-shell X-ray production and ionization cross sections for proton impact", At. Data Nucl. Data Tables, vol. 56, pp. 159-210, 1994.

[39] M. O. Krause, "Atomic Radiative and Radiationless Yields for K and L Shells", J. Phys. Chem. Ref. Data, vol. 8, pp. 307-327, 1979.

[40] M. Batic, M. G. Pia and S. J. Cipolla, "ISICSoo: a class for the calculation of ionisation cross sections from PWBA and ECPSSR theory", submitted to Comp. Phys. Comm., in press, 2011.

[41] R. K. Bock and W. Krischer, "The Data Analysis BriefBook ", Ed. Springer, Berlin, 1998.

[42] G. A. P. Cirrone et al., "A Goodness-of-Fit Statistical Toolkit", IEEE Trans. Nucl. Sci., vol. 51, no. 5, pp. 2056-2063, 2004.

[43] B. Mascialino, A. Pfeiffer, M. G. Pia, A. Ribon, and P. Viarengo, "New developments of the Goodness-of-Fit Statistical Toolkit", IEEE Trans. Nucl. Sci., vol. 53, no. 6, pp. 3834-3841, 2006.

[44] R. A. Fisher, "On the interpretation of $\chi^{2}$ from contingency tables, and the calculation of P", J. Royal Stat. Soc., vol. 85, no. 1, pp. 87-94, 1922.

[45] F. Yates, "Contingency table involving small numbers and the $\chi^{2}$ test", J. Royal Stat. Soc. Suppl., vol. 1, pp. 217-235, 1934.

[46] K. Pearson, "On the $\chi^{2}$ test of Goodness of Fit", Biometrika, vol. 14, no. $1-2$, pp. $186-191,1922$.

[47] M. G. Pia et al., "Evaluation of atomic electron binding energies for Monte Carlo particle transport", IEEE Trans. Nucl. Sci., vol. 58, no. 6 , Dec. 2011.

[48] H. Paul and J. Muhr, "Review of experimental cross sections for Kshell ionization by light ions", Phys. Rep., vol. 135, no. 2, pp. 47-97, 1986.

[49] S. Guatelli, A. Mantero, B. Mascialino, M. G. Pia, and V. Zampichelli, "Validation of Geant4 Atomic Relaxation against the NIST Physical Reference Data", IEEE Trans. Nucl. Sci., vol. 54, no. 3, pp. 594-603, 2007. 